\title{
METODOLOGIA PARA DETECÇÃO ELETROQUÍMICA QUALITATIVA DE COMPOSTOS ORGÂNICOS EM INTERFACES PETRÓLEO/ÁGUA
}

\author{
Roberval A. Silva ${ }^{a}$, Rejane M. P. Silva ${ }^{a}$, Keyla T. Santos ${ }^{a}$, Tatiana S. Araujo ${ }^{a}$, Alexandre C. Alcântara ${ }^{a}$ e Hugo B. Suffredini ${ }^{a}$ *,
}

${ }^{a}$ Centro de Ciências Naturais e Humanas, Universidade Federal do ABC, 09210-580 Santo André - SP, Brasil

Recebido em 20/06/2018; aceito em 27/09/2018; publicado na web em 22/10/2018

\begin{abstract}
METHODOLOGY TO PROMOTE THE QUALITATIVE DETECTION OF ORGANIC COMPOUNDS IN PETROLEUM/WATER INTERFACES. The possibility of detecting some organic compounds directly in a petroleum/water interface was described. Aqueous phase was composed by $1 \times 10^{-3} \mathrm{~mol} \mathrm{~L}^{-1} \mathrm{NaCl}$ solution and the oil phase was carried out using crude oil forming an oil/water interface. Carbon based screen-printed electrodes was used in conjunction with square-wave voltammetry technique, constituting the main tools of this technical note. Petroleum was contaminated with phenol, naphthalene and cyclohexane. The oxidation potentials for these three molecules were $0,72,0,81$ and $0,87 \mathrm{~V}$ vs. Ag, respectively. Phenol was chosen to study the stability of currents during the process. After 15 minutes, the currents of this process were stable. Although the formation of a passivation film, the qualitative detection of the molecules was possible. Finally, the main advantage of this methodology is the possibility of detecting contaminants in oil without the necessity of complicated pre-treatment steps.
\end{abstract}

Keywords: oil/water interfaces; petroleum; qualitative detection; screen-printed electrode.

\section{INTRODUÇÃO}

O petróleo apresenta uma composição química complexa, sendo formado por hidrocarbonetos saturados, insaturados, naftênicos e aromáticos. Em sua constituição, impurezas de compostos sulfurados, nitrogenados, oxigenados, organometálicos, sais dissolvidos, areia e água, são frequentemente identificados. Estas impurezas podem estar presentes em sua composição, ou serem oriundas dos processos produtivos, como a exploração, transporte e refino, elevando o grau de corrosividade dos materiais que constituem os "toners" de armazenamento. ${ }^{1,2}$

A indústria do petróleo dispõe de diferentes tipos de óleos crus para o processamento, tornando necessárias avaliações técnicas e econômicas para que o mercado consumidor possa ser atendido. Estas avaliações, realizadas por procedimentos analíticos, trazem informações das suas características físicas e químicas como o teor de hidrocarbonetos saturados e aromáticos, de resinas e asfaltenos, acidez, teor de contaminantes, densidade, viscosidade, pressão de vapor, ponto de fluidez, entre outros. Estas informações são essenciais para a determinação da qualidade do petróleo bruto. A densidade reflete, em termos médios, o conteúdo de frações leves e pesadas do óleo cru. A acidez, definida como a quantidade de $\mathrm{KOH}$ em miligramas necessários para neutralizar 1 grama de amostra, referidos como número de acidez total, é uma das responsáveis pela corrosão em equipamentos utilizados ao longo dos processos de produção do petróleo. Normalmente, a acidez é provocada pela presença de compostos oxigenados no petróleo cru. ${ }^{2,3}$

Além disso, é interessante para a indústria petrolífera a utilização de metodologias qualitativas simples e de baixo custo para classificar a presença de moléculas em petróleo. Neste contexto, esta nota técnica descreve a utilização de uma nova metodologia qualitativa em que uma gota de petróleo contaminada com diferentes moléculas orgânicas é adicionada a um eletrodo impresso de carbono. Esta gota foi recoberta com solução de $\mathrm{NaCl} 1 \times 10^{-3} \mathrm{~mol} \mathrm{~L}^{-1}$, constituindo, portanto, uma interface do tipo óleo/água. Importante salientar neste ponto que o não é possível realizar medidas eletroquímica

*e-mail: hugo.suffredini@ufabc.edu.br diretamente em petróleo, a não ser que se realizem etapas complicadas de extração ou pré-tratamento das amostras. Neste contexto, surge a importância da nota técnica, pela possibilidade de detectar compostos orgânicos a partir de uma metodologia simples, sem necessidade de pré-tratamento das amostras, conforme descrito a seguir. A Figura 1 mostra uma descrição experimental da metodologia proposta, o que se considera a parte mais importante desta nota técnica:

$\mathrm{O}$ interesse no estudo eletroquímico entre a interface de dois líquidos tem gerado publicações que descrevem em detalhes as aplicações de técnicas eletroquímicas nesse meio anisotrópico, ${ }^{4-11} \mathrm{e}$ trabalhos recentes foram desenvolvidos em interfaces do tipo óleo/ água para estudos de compostos lipossolúveis diretamente em uma fase oleosa sem a necessidade de pré-tratamento das amostras. A fase oleosa, nestes casos, foi composta por biodiesel,,${ }^{12,13}$ óleo de soja, ${ }^{14}$ limoneno, ${ }^{15}$ óleo mineral, ${ }^{16}$ e 1 -undecanol. ${ }^{17}$

Sensores eletroquímicos têm sido utilizados com a finalidade de a caracterizar líquidos complexos, apresentando respostas satisfatórias. ${ }^{4,5}$ Estes estudos podem ser direcionados à caracterização dos constituintes de líquidos, na identificação de contaminantes e no estudo da corrosão, que tais fluidos podem vir a trazer a equipamentos utilizados em seu manejo na indústria. Relacionado ao petróleo, estudos de eletroquímica aplicada a este tipo de constituinte são relativamente comuns na literatura. ${ }^{1,6}$

Durante todas as etapas da cadeia produtiva do petróleo há o frequente contato entre o petróleo cru e a água salina, favorecendo o surgimento de uma interface entre os líquidos imiscíveis, em que podem ser aplicadas técnicas eletroquímicas. ${ }^{6}$

Neste contexto, estudos eletroquímicos em interfaces do tipo petróleo/água permitem detectar contaminantes e, desta forma, estudar o controle da corrosão ou de contaminantes no petróleo. Este trabalho visa estudar uma interface modelo do tipo petróleo/água para a detecção de alguns compostos orgânicos escolhidos, como fenol, ciclohexano e naftaleno, diretamente na gota de petróleo.

Além das questões de equilíbrio e formação de partição, questões como adsorção de produtos podem causar diminuição do sinal analítico. As anisotropias causadas pela diferença de potencial químico causado pela formação de interfaces podem ser descritas conforme itens a seguir: ${ }^{21}$ 


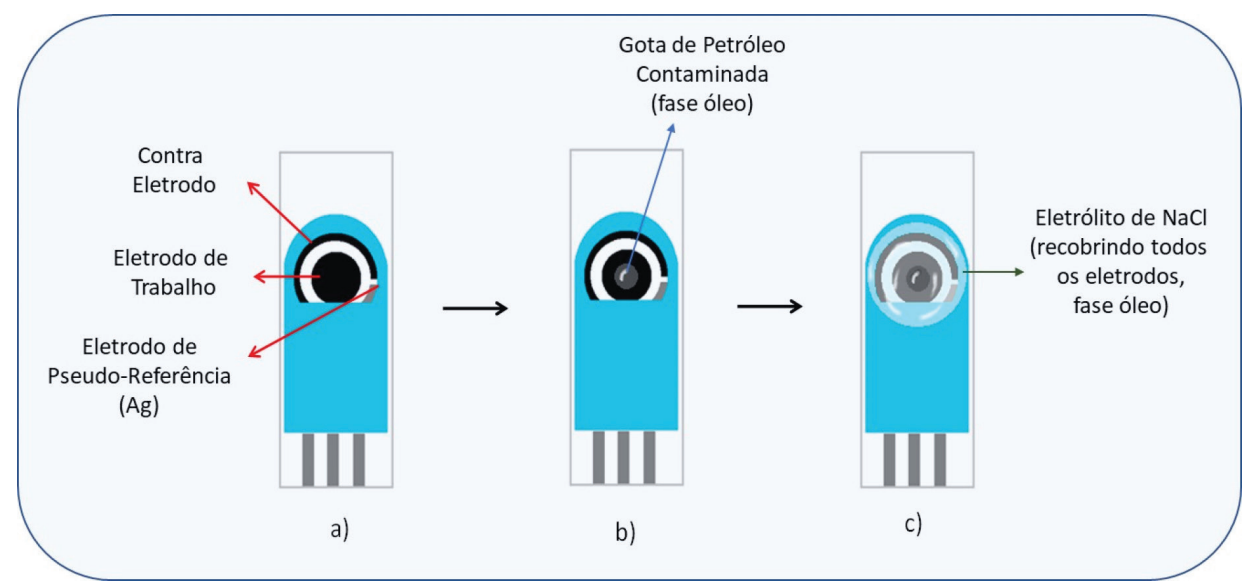

Figura 1. Descrição resumida da técnica experimental proposta, mostrando: a) célula eletroquímica limpa, mostrando a descrição dos eletrodos; b) eletrodo de trabalho com adição da fase oleosa (petróleo); c) adição do eletrólito de suporte ( $\mathrm{NaCl}$ ) sobre a gota de óleo recobrindo todo o eletrodo

- Transferência de um íon "M" com carga “Z” diretamente da fase água para a óleo, ou da fase óleo para a água, representada de maneira simplificada pela reação no equilíbrio:

$$
\mathrm{M}_{\text {(fase água) }}^{\mathrm{Z}} \rightleftarrows \mathrm{M}_{\text {(fase óleo) }}^{\mathrm{Z}}
$$

- Transferência de elétrons entre pares redox do tipo $\mathrm{O}_{1} / \mathrm{R}_{1}$ na fase água e $\mathrm{O}_{2} / \mathrm{R}_{2}$ na fase óleo:

$$
\mathrm{O}_{(1, \text { fase água) }}+\mathrm{R}_{(2, \text { fase óleo })} \rightleftarrows \mathrm{O}_{(2, \text { fase água })}+\mathrm{R}_{(1, \text { fase óleo })}
$$

Estas representações simplificadas, na realidade, podem ser seguidas por processos heterogêneos de transferência de carga, de decomposição química, de desproporcionamento, dentre outros processos paralelos, tornando a análise do sistema mais complicada. No caso do fenol, a reação é acompanhada da formação de um filme de passivação, além dos processos descitos na Eq. 2.

A intensidade do campo elétrico difere de zero na região interfacial entre água e óleo vegetal e a diferença de potencial " $\Delta \phi^{\text {total }}$ " pode ser representada como:

$$
\Delta \phi^{\text {(total })}=\Delta \phi^{\text {(água) }}-\Delta \phi^{\text {(óleo) }}
$$

A condição de equilíbrio para o íon na interface entre dois líquidos imiscíveis pode ser representada a partir de seus respectivos potenciais eletroquímicos $(\bar{\mu})$ :

$$
\bar{\mu}_{M}^{(a g u a)}=\bar{\mu}_{M}^{(\text {oleo })}
$$

que resultam em suas respectivas expressões de potencial eletroquímico expandidas:

$$
\begin{aligned}
& \bar{\mu}_{M}^{(\text {óleo })}=\bar{\mu}_{M}^{0,(\text { óleo })}+R T \ln a_{M}^{(\text {óleo })}+z F \phi^{(\text {óleo })} \\
& \bar{\mu}_{M}^{(\text {aggua })}=\bar{\mu}_{M}^{0,(\text { água })}+R T \ln a_{M}^{(\dot{a g} z u a)}+z F \phi^{(\dot{a} g u a)}
\end{aligned}
$$

onde $\bar{\mu}^{0}$ representa os potenciais eletroquímicos padrões, “ $\phi$ ” representa a diferença de potencial gerada nas proximidades das fases óleo e água e " $a_{\mathrm{M}}$ " é a atividade dos íons.

Combinando-se as equações 3,5 e 6 , é possível conhecer a diferença de potencial no equilíbrio entre as duas fases:

$$
\Delta \phi^{(\text {total })}=\Delta \phi^{0,(\text { total })}+(R T / z F) \ln \left(a_{M}^{(\text {óleo })} / a_{M}^{(\text {água })}\right)
$$

Assim, a diferença de potencial galvânico entre as duas fases pode ser escrita como:

$$
\Delta \phi_{M}^{(\text {Total })}=-\left(\mu_{M}^{(0, \text { agua })}+\mu_{M}^{(0, \text { óleo })}\right) / z F
$$

Desta forma, após o entendimento dos processos de formação da partição no equilíbrio e da movimentação de massa e carga das espécies envolvidas na interface, novas e interessantes aplicações podem ser realizadas, conforme comentado anteriormente. A aplicação desta proposta passa por esta questão de formação das partições e de processos que podem prejudicas o sinal analítico, como adsorção de produtos de oxidação/redução (como é o caso do fenol), dentre outros parâmetros, como aumento da resistividade do material e ativação do eletrodo. No entanto, a avaliação qualitativa pode ser realizada, embora existam todas estas barreiras a serem vencidas.

\section{PARTE EXPERIMENTAL}

Nos testes realizados, utilizou-se um sistema composto um potenciostato Autolab PGStat ${ }^{\circledR}$, conector e eletrodo de carbono impresso da marca DropSens ${ }^{\circledR}$ modelo 110 . O eletrodo de carbono impresso é uma célula eletroquímica completa miniaturizada, com dimensões $3,4 \times 1,0 \times 0,05 \mathrm{~cm}$. Nessa estrutura tem-se a presença de um contra eletrodo e um eletrodo de trabalho compostos por carbono e de um eletrodo de pseudo-referência composto por prata metálica. Esse tipo de célula tem sido muito utilizado nos últimos anos devido ao seu baixo custo de produção e a redução de reagentes e amostras utilizados. Além disso, por ser descartável, minimiza os riscos de contaminação. ${ }^{19}$ Inicialmente adicinou-se, com uma micro-pipeta, $1 \mu \mathrm{L}$ da fase oleosa no centro do eletrodo de trabalho. Em seguida, adicionou-se o eletrólito de suporte $\mathrm{NaCl} 1 \times 10^{-3} \mathrm{~mol} \mathrm{~L}^{-1}$, recobrindo toda a extensão da célula eletroquímica. A escolha do eletrólito de suporte basseado em $\mathrm{NaCl}$ se justifica pelo fato de ser o sal presente em maior quantidade na água do mar. As concentrações salinas são bastante superiores na água do mar, mas este problema pode ser contornado com o tratamento e diluição da amostra, conforme protocolo a ser estabelecido em estudo analítico completo. Desta forma, a concentração de $1 \times 10^{-3}$ foi escolhida como ponto de partida. A esse sistema de interface, formado pela superfície do eletrodo, fase óleo e fase aquosa, aplicou-se a voltametria de onda quadrada nas seguintes condições de $\mathrm{f}=30 \mathrm{~s}^{-1} ; \mathrm{a}=30 \mathrm{mV} ; \Delta \mathrm{Es}=2 \mathrm{mV}$. Os testes foram repetidos utilizando como fase oleosa o petróleo bruto pré contaminado ora com fenol, ora com cliclohexano e ora com naftaleno, ambos com concentração de de $1 \times 10^{-2} \mathrm{~mol} \mathrm{~L}^{-1}$ em petróleo. Estas concentrações também foram escolhidas como ponto de partida e devem ser melhor estudadas em uma avaliação analítica mais completa. 


\section{RESULTADOS E DISCUSSÃO}

Depois de montada a metodologia descrita nesta nota técnica, foi possível realizar a detecção de moléculas orgânicas introduzidas artificialmente no petróleo diretamente em uma interface do tipo petróleo/água, conforme mostrado na Figura 2.

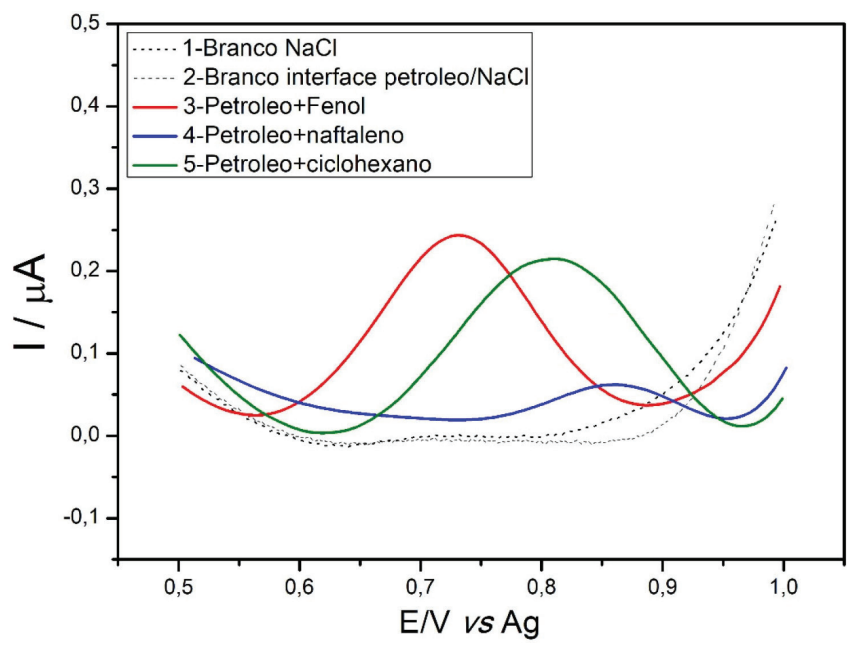

Figura 2. Voltametrias de onda quadrada para as respostas obtidas na fase aquosa $\mathrm{NaCl} 1 \times 10^{-3} \mathrm{~mol} \mathrm{~L}^{-1}$ (curva 1),na interface petróleo/ $\mathrm{NaCl}$ $1 \times 10^{-3} \mathrm{~mol} \mathrm{~L}^{-1}$ (curva 2) e no sistema interfacial petróleo/eletrólito de suporte $\left(\mathrm{NaCl} 1 \times 10^{-3} \mathrm{~mol} \mathrm{~L}^{-1}\right)$ contendo os compostos orgânicos : fenol (curva 3), naftaleno (curva 4) e ciclohexano (curva 5) ambos os analitos foram preparados em petróleo cru, com concentrações iguais a $1 \times 10^{-2} \mathrm{~mol} \mathrm{~L}^{-1}, f=30 \mathrm{~s}^{-1}$, $a=30 \mathrm{mV} e \Delta E s=2 \mathrm{mV}$

Observa-se que a detecção de fenol, naftaleno e ciclohexano foi possível diretamente em uma interface do tipo óleo/água, em potenciais de oxidação diferentes $(0,72,0,81$ e $0,87 \mathrm{~V} v s$ Ag para fenol, naftaleno e ciclohexano, respectivamente). Importante salientar aqui que a detecção das moléculas ocorreu nos instantes iniciais da medida, para evitar problemas com adsorção de orgânicos ou produtos de reação na superfície do eletrodo de trabalho. Observou-se ainda nos perfis voltamétricos apresentados na Figura 2 que não ocorrem processos de oxidação importantes no eletrólito puro (curva 1) e no petróleo bruto (curva 2) na janela de potencial adotada. A pequena protuberância dos brancos é referente à inerente corrente capacitiva dos eletrodos de carbono, que apresentaram resposta não significativa para os propósitos deste trabalho. A Figura 2 representa apenas um exemplo do que a metodologia propõe, enfatizando que a importância da nota técnica está relacionada à montagem experimental apresentada na Figura 1

Como o perfil voltamétrico de oxidação do fenol foi o mais bem definido, escolheu-se esta molécula para as análises posteriores. Além de tudo, o fenol conhecidamente apresenta problemas de passivação de eletrodo, pela formação de filmes poliméricos ou adsorção da molécula original. Desta forma, acredita-se que seja um bom candidato para as análises qualitativas. Estudos de detecção de fenol e derivados em eletrodos impressos ou modificados foram recentemente apresentados nos trabalhos de Talarico et al. ${ }^{20}$ Arabali et al. ${ }^{21}$ Piovesan et al. ${ }^{22}$ e Yaman et al.,$^{23}$ todos em meios aquosos. Em praticamente todos os casos, o fenol adsorve fortemente na superfície dos materiais, causando perda de sensibilidade nas análises eletroquímicas. De fato, a Figura 3 mostra que ocorre uma diminuição constate na corrente de pico de oxidação do fenol, tornando útil que se realize a análise qualitativa em instantes iniciais, caso o objetivo principal seja o de detectar esta molécula.

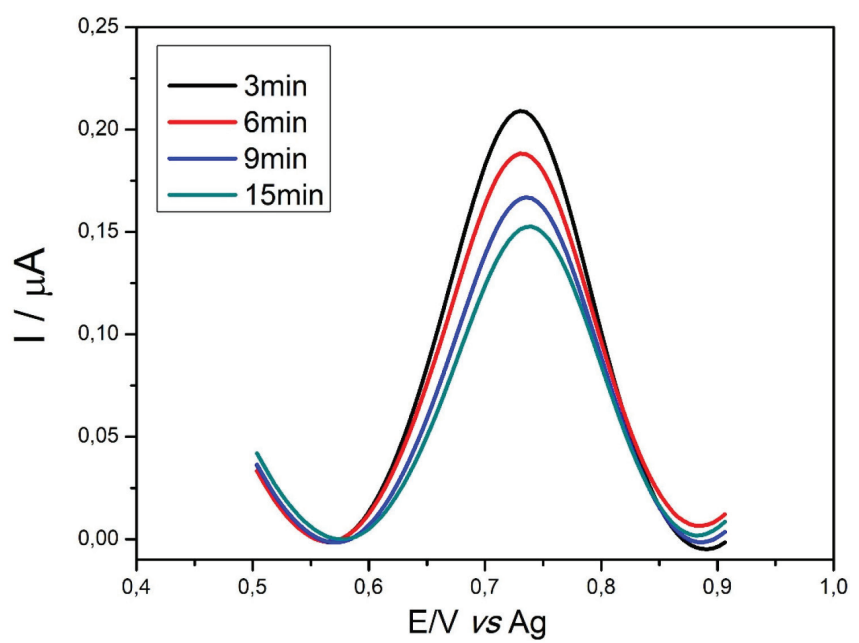

Figura 3. Voltametrias de onda quadrada representando os picos de oxidação do fenol, com o tempo de varredura variando de $3 \mathrm{em} 3$ minutos. Demais condições: $f=30 \mathrm{~s}^{-1} ; a=30 \mathrm{mV}$ e $\Delta E S=2 \mathrm{mV}$

De acordo com a Figura 3, é possível observar que ocorre a diminuição da corrente em intervalos de 3 a 15 minutos. Neste caso, o fenol causa a formação de um filme de passivação na superfície eletródica, com consequente diminuição da resposta de corrente. ${ }^{24}$

Um mecanismo de reação para a reação de oxidação do fenol pode ser encontrado a seguir, na Figura 4:

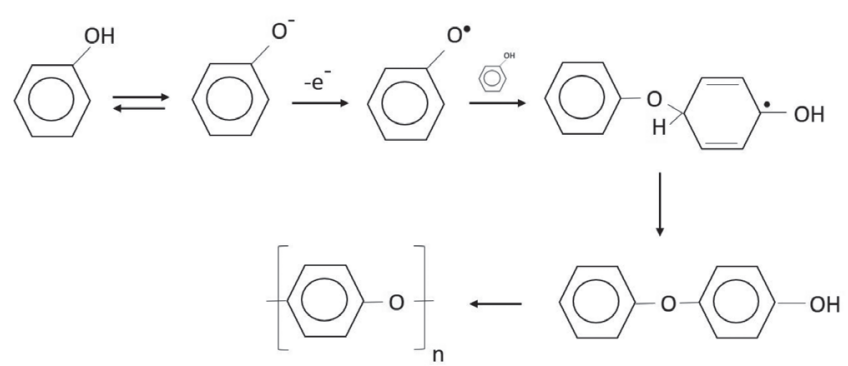

Figura 4. Mecanismo de oxidação do Fenol para meios com pKa <9,89, com formação de produtos de polimerização a partir da formação de radicais fenoxil. Os testes deste trabalho foram realizados em meio neutro

Por meio do gráfico da Figura 5, foi possível constatar a corrente de oxidação do fenol diretamente na interface petróleo/água, atingiu o equilíbrio depois de aproximadamente 15 minutos.

Apesar da produção de um filme de passivação, causando diminuição nas correntes de oxidação, verificou-se que após 15 minutos não ocorreram variações significativas de corrente, o que indica um equilíbrio entre filme de passivação e oxidação da molécula de fenol original, conforme mostrado na Figura 5, referente ao monitoramento da corrente de oxidação do fenol em função do tempo. Este fator mostra que, ainda que ocorram problemas causados pela diminuição da corrente, o eletrodo se mantém ativo para a detecção qualitativa nestas condições. Do ponto de vista qualitativo, o eletrodo deve ser utilizado em seus instantes iniciais para que a formação do filme de passivação não influencie a detecção das outras moléculas, como mostrado na Figura 2.

Como ocorrem processos de particionamento entre as fases água e óleo, tempos de estabilização de corrente ocorrem com frequência. Como exemplos, em trabalho publicado por Maynart et al. ${ }^{12}$ que estudaram a oxidação do ferroceno diretamente na interface biodiesel/água utilizando eletrodos de diamante dopado com boro, foram observadas correntes de pico de oxidação relativamente constantes após 15 minutos. Silva et al. ${ }^{13}$ estudaram também o monitoramento da 


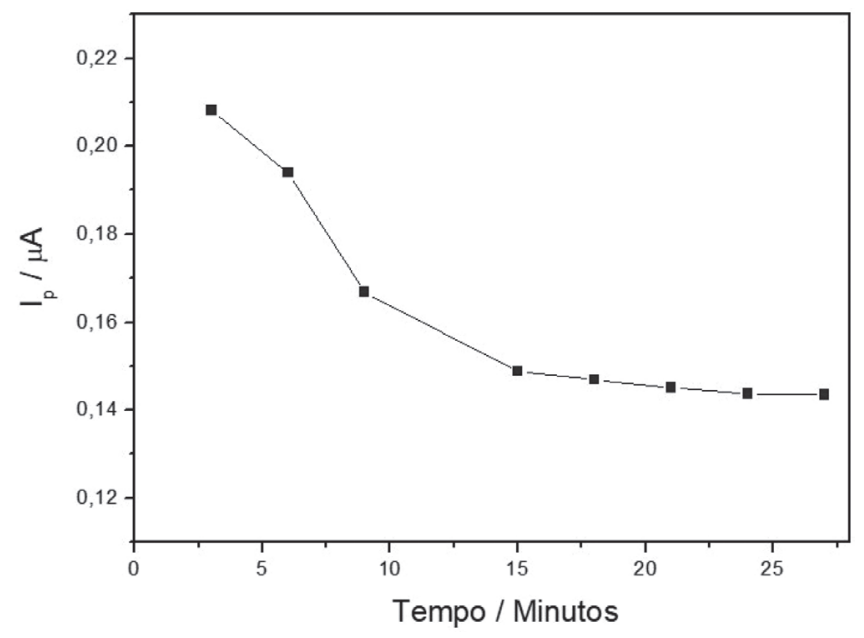

Figura 5. Correntes de pico de oxidação $\left(I_{p}\right)$ mostrando a estabilidade de corrente de oxidação, atingida depois de cerca de 15 minutos de operação

corrente de oxidação do ferroceno na fase aquosa, em uma interface biodiesel/água, na qual ocorria a migração da molécula eletroativa da fase óleo para a fase água, nessa condição o sistema atingiu o equilíbrio após aproximadamente 175 minutos de operação.

\section{CONCLUSÃO}

Nesta nota técnica, procurou-se mostrar que um sistema constituído por eletrodo impresso de carbono pode ser utilizado para a realização de estudos em interfaces água/petróleo, sem a necessidade de pré-tratamento das amostras. Para tanto, uma gota de petróleo (fase óleo) foi inserida na superfície do eletrodo, que posteriormente foi recoberto com solução salina. Este protótipo foi utilizado para a detecção qualitativa de três moléculas orgânicas distintas, mostrando a viabilidade da metodologia, apesar dos mecanismos de oxidação do fenol promoverem a formação de um filme de passivação em meio neutro. Nos estudos com a molécula de fenol, foi possível verificar o comportamento da corrente de oxidação na interface petróleo/água e monitorar o tempo de equilíbrio da molécula na região interfacial, atingido em aproximadamente 15 minutos, mostrando que o eletrodo funciona em instantes iniciais de maneira adequada. Mesmo após 15 minutos e depois de formado um filme de passivação, o sistema detectou o fenol nas condições de estudo.

\section{AGRADECIMENTOS}

FAPESP (procs. 2018/06677-6, 2017/10381-2, 2014/13602-1) e CNPq (proc. 304797/2017-0)

\section{REFERÊNCIAS}

1. Cosultchi, A.; García-Bórquez, A.; Aguilar-Hernandez, J.; Yee-Madeira H.; Reguera, E.; Lara, V. H.; Bosch, P; Surf. Interf. Anal. 2002, 34, 384.

2. Farah, A. M. Em: Petróleo e seua derivados, LTC: Rio de Janeiro, 2013.

3. Farias, R. F. D. Em Introdução à química do petróleo, Editora Ciência Moderna: São Paulo, 2008.

4. Koryta, J.; Electrochim. Acta 1988, 33, 189.

5. Vanýsek, P.; Electrochim. Acta 1998, 40, 2841.

6. Banks, C. E.; Davies, T. J.; Evans, R. G.; Hignett, G.; Wain, A. J.; Lawrence, N. S.; Wadhawan, J. D.; Marken, F.; Compton, R. G.; Phys. Chem. Chem. Phys 2003, 5, 4053.

7. Girault, H. H.; Em Modern Aspects of Electrochemistry; Bockris, J. O. M., Conway, B. E., White, R. E., eds.; Springer: Boston, 1993.

8. Gulaboski, R.; Galland, A.; Bouchard, G.; Caban, K.; Kretschmer, A.; Carrupt, P. A.; Stojek, Z.; Girault, H. H.; Scholz, F.; J. Phys. Chem B 2004, 108, 4565.

9. Kachoosangi, R. T.; Xiao, L.; Wildgoose, G. G.; Marken, F. ; Bulman Page, P. C.; Compton, R. G.; J. Phys. Chem. C 2007, 111, 18353.

10. Komorsky-Lovrić, Š.; Lovrić, M.; Scholz, F.; J. Electroanal. Chem. 2001, 508, 129.

11. Shao, Y.; Osborne, M. D.; Girault, H. H.; J. Electroanal. Chem. 1991, 318, 101.

12. Maynart, M. C.; Ferreira, R. Q.; Suffredini, H. B.; Electroanalysis 2010, 23,330 .

13. Silva, R. M. P.; Maynart, M. C.; Suffredini, H. B.; Ionics 2014, 20, 1183.

14. Maynart, M. C.; Silva, R. M. P.; Suffredini, H. B.; Electroanalysis 2013, 25,1541 .

15. Oliveira, E. S.; Fiorito, P. A.; Suffredini, H. B.; Electroanalysis 2014, 26, 1660 .

16. Silva, R. M. P.; Silva, G. D.; Coutinho-Neto, M. D.; Suffredini, H. B.; Electrochim. Acta 2016, 212, 195.

17. Silva, R. M. P.; Lima, L. C.; Gaubeur, I.; Suffredini, H. B.; Electroanalysis 2017, 29, 259.

18. Suffredini, H. B.; Estudo da Viabilidade da Utilização de Interfaces Líquido/Líquido em Dispositivos Eletroquímicos, Projeto Fapesp 2018/06677-6, 2018.

19. Cui, G.; Yoo, J. H.; Lee, J. S.; Yoo, J.; Uhm, J. H.; Cha, G. S. ; Nam, H.; Analyst 2001, 126, 1399.

20. Talarico, D. ; Arduini, F.; Constantino, A.; Del Carlo, M.; Compagnone, D.; Moscone, D.; Palleschi, G.; Electrochem. Commun. 2015, 60, 78.

21. Arabali, V.; Ebrahimi, M.; Gheibi, S.; Khaleghi, F.; Bijad, M.; Rudbaraki, A.; Abbasghorbani, M.; Ganjali, M. R.; Food Anal. Methods 2016, $9,1763$.

22. Piovesan, J. V.; Jost, C. L.; Spinelli, A.; Sens. Actuators, B: Chem. 2015, 216, 192.

23. Yaman, Y. T.; Abaci, S.; Sensors 2016, 16, 756.

24. Yang, X.; Kirsch, J.; Fergus, J.; Electrochim. Acta 2013, 94, 259. 